\title{
Two fascinating X-ray to optical cross-correlation functions
}

\author{
Martin Durant* \\ I. A. C., Tenerife, Spain \\ E-mail: duranteiac.es

\section{Poshak Gandhi} \\ RIKEN Cosmic Radiation Lab, Japan \\ E-mail: pg@crab.riken.jp
}

\begin{abstract}
We have measured the X-ray/optical cross-correlation functions (CCFs) of the two black hole binary systems SWIFT J1753.5-0127 and GX 339-4 using ULTRACAM and RXTE. We find each one is unique and different from the one such measurement in the literature, for XTE J1118+480. Our CCFs are inconsistent with reprocessing, and suggest a mechanism which regulates emission between the different spectral bands, possibly of magnetic origin.
\end{abstract}

VII Microquasar Workshop: Microquasars and Beyond September 1-5 2008

Foca, Izmir, Turkey

\footnotetext{
* Speaker.
} 
This contribution is a summary of the results in the papers Durant et al. (2008a, ApJ, 682, L45) and Gandhi et al. (2008, MNRAS, 390, L29), and a comparison between them. A general public version of this work will also appear as an ESO press release ${ }^{1}$.

Further details of the observations campaigns is published in Durant et al. (2008b) for the case of SWIFT J1753.5-0127 and Gandhi et al. (2009 in prep.) for the case of GX 339-4.

\section{Introduction}

Binary accreting systems are dynamic by their very nature. Each system contains a variety of emitting regions of different energy scales (a donor star, a matter stream, an impact spot, an multitemperature accretion disc, a halo, jets...), and many characteristic time-scales may exist (e.g., the orbital period, Keplerian periods, light-crossing times, acoustic resonances...). These all interact with one-another, and furthermore, the whole accretion process is known to be unstable and involve hysteresis in changing between various modes or states. There is much discussion on the long timescale evolution of microquasar systems in these proceedings. Any successful model of accretion and microquasars must explicitly consider dynamics in the system.

Being such a rich source of timing phenomenology, microquasars have been studied for a long time at high time resolutions, but almost exclusively in the X-ray band. X-ray observations have intrinsically good time resolution (milliseconds), but typically low count rates. It is only relatively recently that high time resolution observations have become possible in the optical band (e.g., OPTIMA, Kanbach et al. 2001). Of great interest is the comparison between the different observation bands: when you sample energies as different as X-rays and optical, you can infer how different components of the accreting system affect one another. This is the critical ingredient that dynamic models must aim to explain.

Particularly important for our observations campaign, are the two instruments Rossi X-ray Timing Explorer (RXTE) and ULTRACAM (Dhillon et al. 2007), they provide both the time resolution and some spectral information with scheduling flexible enough to be able to perform simultaneous observations.

Kanbach et al. (2001) were the first to derive a detailed cross-correlation function between X-ray and optical emission, for the black hole system XTE J1118+480. They were surprised to find that, aside from the main positive correlation for optical-lagging-X-rays, there was also an anti-correlation component for optical-leading-X-rays. The first could easily be understood as reprocessing, that optically thick material in the disc absorbs X-ray radiation and re-emits it at a wavelength corresponding to typical temperatures there: predominantly in the optical. In that scenario, the optical must always come second, with a lag corresponding to the light travel time and reprocessing time. Such an idea is used in echo timings, to map the motion of the binary companion, for example. The anti-correlation component is harder to explain, however.

\section{Cross-correlations}

We observed two black hole accreting binary systems, SWIFT J1753.5-0127 and GX 339-4 with ULTRACAM and RXTE simultaneously in June 2007. From the resulting light-curves, we

\footnotetext{
${ }^{1}$ see http://www-xray.ast.cam.ac.uk/ pp/flickering
} 


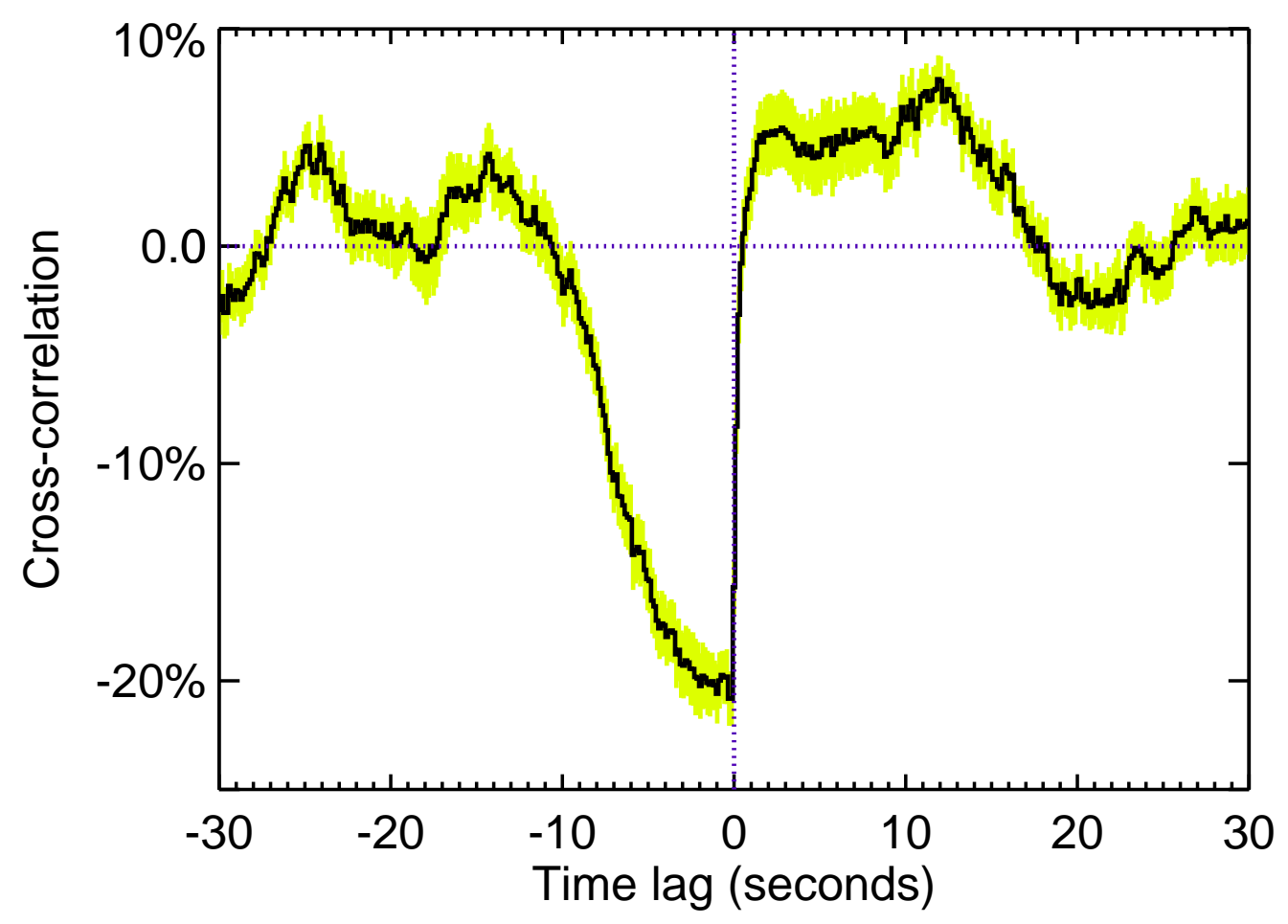

Figure 1: Cross-correlation function for SWIFT J1753.5-0127. Negative lags imply optical light arriving before X-ray, positive lags imply optical light arriving after X-rays. The shaded zone indicates the uncertainty on the function.

calculated the X-ray/optical cross-correlation functions. Both sources were in the "low-hard" X-ray state at the time of observation.

One point, that cannot be made strongly enough, is that these are average relationships. None of the light-curves is made up of identifiable isolated flares, and the CCF does not therefore describe a behaviour that happens for every flare, only a statistical likelihood. Whether the light-curves can or cannot be described as a superposition of flares is not clear.

\subsection{SWIFT J1753.5-0127}

Figure 1 shows the CCF for SWIFT J1753.5-0127. The units here are different than those in Durant et al. (2008a) in order to be be consistent with Figure 2, below. The principal features that can be seen are:- a strong, broad anti-correlation between the wave-bands, when the optical arrives up to $10 \mathrm{~s}$ before the X-rays; a very rapid swing at zero lag (i.e., arriving simultaneously); a weaker positive correlation for optical later than X-rays, up to $15 \mathrm{~s}$ broad and fairly flat; and some further oscillation behaviour further from zero lag in both directions, which looks rather like noise.

\subsection{GX 339-4}

The CCF for GX 339-4 (see Figure 2) is markedly different from that for SWIFT J1753.5-0127 (Figure 1). There is a broad and weak anti-correlation centred at $-4 \mathrm{~s}$ (optical arriving before X- 


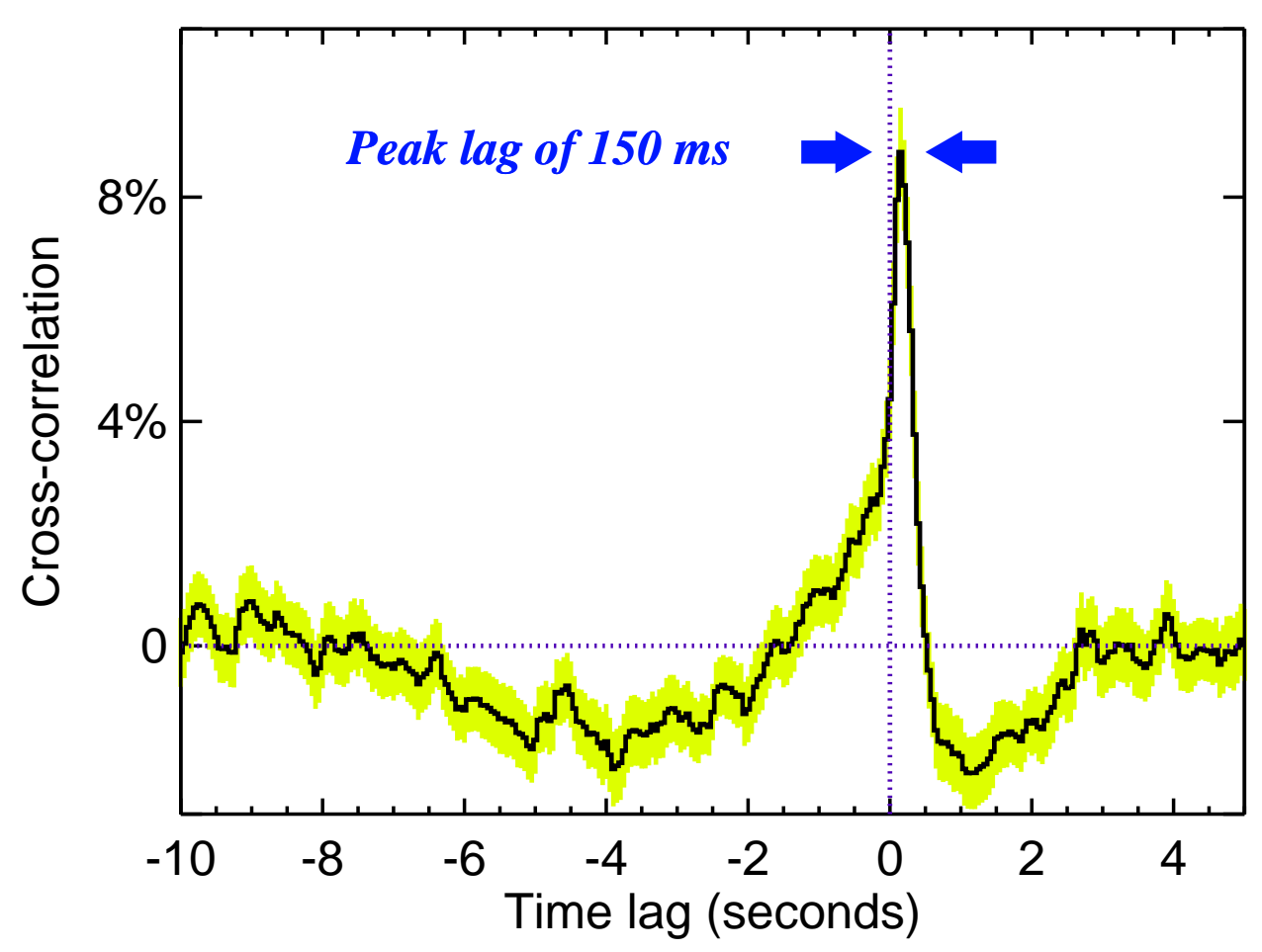

Figure 2: Cross-correlation function for GX 339-4, displayed as in Figure 1.

rays), a gradual rise to a stronger correlation peak located just after zero lag at $+150 \mathrm{~ms}$, a sharp decline and a further broad, weak anti-correlation centred at $+1 \mathrm{~s}$. An alternative interpretation of the graph, is that there are two components, one very broad anti-correlation centred at around $-3 \mathrm{~s}$, and the sharp positive correlation at $+150 \mathrm{~ms}$. Importantly, the positive feature does not have the shape characteristic of reprocessing, which would be a sharp rise near to, but after, zero lag, and a less sharp decline.

\section{Interpretation}

There are now a total of three well-determined X-ray/optical CCFs, for XTE J1118+480, and now SWIFT J1753.5-0127 and GX 339-4. All three are unique. For the latter two, presented here, there is little evidence for a reprocessing component, which would rise quickly near zero and fall less quickly towards positive lags. Furthermore, the optical auto-correlartion functions are narrower in each case than for the corresponding X-rays, which again is a surprise. The features that are seen are not consistent with the reprocessing idea. Reprocessing probably does still occur, but at a rather low level.

For both sources, it may be possible to decompose the CCFs into constituent components, by the Fourier cross-spectrum or other methods. For example, the CCF for SWIFT J1753.5-0127 (Figure 1) may have a reasonably strong reprocessing-like peak for positive lags, if the anticorrelation feature is symmetric about zero, reaching to about $+10 \mathrm{~s}$. Similarly, in Figure 2 one 
may see just two components, one sharp positive response near zero and on very broad and shallow negative signal centre at near $-3 \mathrm{~s}$.

Rapid variability as seen here must happen close to the accreting black hole of each system, and the optical and X-ray emission must have a common origin. Since anti-correlation is an important feature of out CCFs, particularly for SWIFT J1753.5-0127, either a mechanism which can channel energy into one or another emitting mode, or a direct competition between the two modes is required. Although the radiation process responsible for optical is unknown, it is reasonable to assume that it is magnetic in nature (synchro-cyclotron), since simple thermal reprocessing is already excluded, and bremsstrahlung does not fit the observed spectra. Likewise, for a hard power-law X-ray spectrum to high energies $(>50 \mathrm{keV})$, comptonisation by a high-energy medium is likely. In this case, the optical luminosity would depend on the surface area and magnetic field strength of the optically emitting area (presumably the disc) and the X-ray luminosity would depend on the particle energy content, size and seed photon density of X-ray emitting region (possibly a corona, inner under-dense zone or the jets). Connection between the two can be made by the flow of particles, evaporation and condensation to and from the disc, and by magnetic fields, which are produced in the disc and dissipated outside the disc.

One possible scenario that may simply explain the different complex CCFs is the magnetic reservoir model of Malzac et al. (2004), where energy can be stored in the magnetic field, which channels them to different emission regimes. Thus anti-correlations and differential-like relationships can be observed between the emitting modes, and a range of cross-correlation behaviours created. This model was originally developed for the specific case of XTE J1118+480. In our view, such an analysis, explicitly considering the dynamics of the sistem as a base ingredient, is the only way to proceed in the understanding of microquasar systems in general.

In summary, we present two optical/X-ray CCFs for the black hole systems SWIFT J1753.5-0127 and GX 339-4, bringing the total number of well-measured CCFs to three. Each so far is unique, and to our surprise, we find that reprocessing is not a key player in the relationship between optical and X-ray emission. These functions give us imporant clues to the behaviour of the different emitting components very close to the black hole, and detailed dynamical modelling, as well as studying the evolution of the CCFs, will finally begin to shed light on the conditions and physical processes at extreme gravitational field strengths.

\section{References}

[1] Dhillon, V. S., Marsh, T. R., Stevenson, M. J., Atkinson, D. C., Kerry, P., Peacocke, P. T., Vick, A. J. A., Beard, S. M., Ives, D. J., Lunney, D. W., McLay, S. A., Tierney, C. J., Kelly, J., Littlefair, S. P., Nicholson, R., Pashley, R., Harlaftis, E. T., O’Brien, K., ULTRACAM: an ultrafast, triple-beam CCD camera for high-speed astrophysics, 2007, MNRAS, 378, 825 [arXiv: 0704 .2557]

[2] Durant, M., Gandhi, P., Shahbaz, T., Fabian, A. P., Miller, J., Dhillon, V. S., Marsh, Tom R., SWIFT J1753.5-0127: A Surprising Optical/X-Ray Cross-Correlation Function, 2008a, ApJ, 682, L45 [arxiv:0806.2530]

[3] Durant, M., Gandhi, P., Shahbaz, T., Peralta, H. H., Dhillon, V. S., Multiwavelength spectral and high time resolution observations of SWIFT J1753.5-0127: new activity?, 2008b, MNRAS accepted, arxiv:0810.1141 
[4] Gandhi, P., Makishima, K., Durant, M., Fabian, A. C., Dhillon, V. S., Marsh, T. R., Miller, J. M., Shahbaz, T., Spruit, H. C., Rapid optical and X-ray timing observations of GX 339-4: flux correlations at the onset of a low/hard state, 2008, MNRAS, 390, L29 [arxiv: 0807.1529]

[5] Kanbach, G., Straubmeier, C., Spruit, H. C., Belloni, T., Correlated fast X-ray and optical variability in the black-hole candidate XTE J1118+480, 2001, Nature, 414, 180

[6] Malzac, J., Merloni, A., Fabian, A. C., Jet-disc coupling through a common energy reservoir in the black hole XTE J1118+480, 2004, MNRAS, 351, 253 